\title{
Dopaminergic Striatal Innervation Predicts Interlimb Transfer of a Visuomotor Skill
}

\author{
Ioannis U. Isaias, ${ }^{1,2,3}$ Clara Moisello, ${ }^{4}$ Giorgio Marotta, ${ }^{5}$ Mauro Schiavella, ${ }^{2}$ Margherita Canesi, ${ }^{2}$ Bernardo Perfetti, ${ }^{4}$ \\ Paolo Cavallari, ${ }^{1}$ Gianni Pezzoli, ${ }^{2}$ and M. Felice Ghilardi ${ }^{4}$ \\ ${ }^{1}$ Università degli Studi di Milano, Dipartimento di Fisiologia Umana, 20133 Milano, Italy, ${ }^{2}$ Centro per la Malattia di Parkinson e i Disturbi del Movimento, \\ Istituti Clinici di Perfezionamento, 20126 Milano, Italy, ${ }^{3}$ Universitätsklinik Würzburg, Neurologische Klinik und Poliklinik, 97080 Würzburg, Germany, \\ ${ }^{4}$ CUNY Medical School, Department of Physiology and Pharmacology, New York, New York 10031, and 5Fondazione IRCCS Ca' Granda Ospedale Maggiore \\ Policlinico, Dipartimento di Medicina Nucleare, 20122 Milano, Italy
}

We investigated whether dopamine influences the rate of adaptation to a visuomotor distortion and the transfer of this learning from the right to the left limb in human subjects. We thus studied patients with Parkinson disease as a putative in vivo model of dopaminergic denervation. Despite normal adaptation rates, patients showed a reduced transfer compared with age-matched healthy controls. The magnitude of the transfer, but not of the adaptation rate, was positively predicted by the values of dopamine-transporter binding of the right caudate and putamen. We conclude that striatal dopaminergic activity plays an important role in the transfer of visuomotor skills.

\section{Introduction}

Motor learning relies on the intact function of dopaminergic transmission (Knowlton et al., 1996; Seidler et al., 2006; Seidler and Noll, 2008; Karabanov et al., 2010). In particular, dopamine neurons play an important role in coding reinforcement prediction errors, a key signal in many learning models (Sutton and Barto, 1998; Maia, 2009). Prediction errors are used to learn the values of states, state-action pairs, or both, which are then used to select optimal actions (Sutton and Barto, 1998). At the cellular and synaptic level, such learning is thought to occur through long-term changes in synaptic strength at striatal synapses, with dopamine release as an essential signaling event starting the process (Montague et al., 2004; Calabresi et al., 2007).

Adaptation of reaching movements to visuomotor rotation is a type of learning that can take place implicitly. During this learning process, a new internal model or memory is gradually formed, while the discrepancy between the desired and the executed trajectory gradually diminished (Krakauer, 2009). Several studies have suggested a right-hemisphere dominance in the acquisition of this motor skill (Ghilardi et al., 2000; Huber et al., 2004), but the role of dopamine and its basal ganglia innervation in visuomotor adaptation has been poorly investigated. Previous studies have shown that adaptation but not retention is normal in pa-

\footnotetext{
Received July 13, 2011; revised Aug. 16, 2011; accepted Aug. 18, 2011.

Author contributions: I.U.I., C.M., and M.F.G. designed research; I.U.I., G.M., M.S., and M.C. performed research; I.U.I., C.M., G.M., M.S., M.C., B.P., P.C., G.P., and M.F.G. analyzed data; I.U.I., C.M., G.M., B.P., P.C., G.P., and M.F.G. wrote the paper.

This study was supported by the Grigioni Foundation for Parkinson Disease (to I.U.I.), the National Parkinson's Foundation (to G.M.F.), and NIH Grant NS054864 (to G.M.F.). We thank Prof. Jens Volkmann (Department of Neurology, University of Würzburg, Würzburg, Germany) for critically reading this manuscript.

The authors declare no competing financial interests.

Correspondence should be addressed to Dr. Ioannis U. Isaias, Dipartimento di Fisiologia Umana, Università degli Studi, 20133 Milano, Italy. E-mail: iuisaias@yahoo.it.

DOI:10.1523/JNEUROSCI.3583-11.2011

Copyright $\odot 2011$ the authors $\quad 0270-6474 / 11 / 3114458-05 \$ 15.00 / 0$
}

tients with Parkinson disease (PD) (Marinelli et al., 2009; Bédard and Sanes, 2011; Venkatakrishnan et al., 2011), but have not addressed the role of dopamine in this task. However, the existing evidence in normal subjects suggests that the striatum plays an important role in the formation and retrieval of this motor skill (Ghilardi et al., 2000; Seidler et al., 2006; Seidler and Noll, 2008).

When learning is accomplished with one limb through task repetition, the ability to perform the same task with the opposite, untrained limb can also improve. This process is referred to interlimb transfer and has been shown to apply to visuomotor adaptation (Sainburg and Wang, 2002). Interlimb transfer implies that the limb-specific motor memory, stored during adaptation, can be retrieved and applied to the other limb with different dynamic characteristics. As reported in several studies, the corpus callosum is likely involved in this process (for review, see Halsband and Lange, 2006). However, given their important role in motor learning, dopamine and the striatum could be also involved in such a skill, but their function has not been directly assessed.

The aim of this study is to determine whether the adaptation to visuomotor rotation and its transfer to the untrained hand depend on dopamine levels. Thus, we investigated the time course of adaptation to a rotated display with the right hand and its transfer to the left hand in drug-naive patients with PD and in age-matched controls. We then correlated the indices of learning and transfer to dopaminergic innervation as measured by $\left[{ }^{123} \mathrm{I}\right] N$ - $\omega$-fluoropropyl$2 \beta$-carbomethoxy- $3 \beta$-(4-iodophenyl)tropane (FP-CIT) and singlephoton computed tomography (SPECT) (Isaias et al., 2007).

\section{Materials and Methods}

Subjects. We tested 11 patients with PD (five males; median age, 52 years; range, 38-66 years) and a control group of 10 neurologically intact adults (three males; median age, 52 years; range, $42-79$ years). Median age of $\mathrm{PD}$ patients at motor symptoms onset was 47 years (33-64 years). 
All subjects were right-handed, as assessed by a modified Edinburgh handedness inventory.

The diagnosis of PD was made according to the UK Parkinson Disease Brain Bank criteria and patients evaluated with the Unified Parkinson Disease Rating Scale motor part (UPDRS-III). Two additional UPDRS sub-subscores were calculated (Isaias et al., 2007). Median UPDRS-III score was 12 (range, 6-20). Median akinetic-rigid score (UPDRS-AK) was 5 (range, 2-11) and median UPDRS tremor score (UPDRS-T) was 1 (range, $0-2$ ).

Clinical inclusion criteria for subjects with PD were as follows: (1) UPDRS part I score of 0; (2) UPDRS-T score $<3$; (3) disease duration $<5$ years; (4) Hoehn and Yahr scale, stage 2; (5) drug naive (patients had never been treated with any antiparkinsonian drugs); (6) no psychiatric disorders or other neurological diseases other than PD; and (7) absence of any signs indicative for atypical parkinsonism (e.g., gaze abnormalities, autonomic dysfunction, psychiatric disturbances, etc.).

All subjects (patients and controls) had no cognitive decline as well as no deficit in visual attention, task switching, memory, or learning strategies, as assessed by the Mini-Mental State examination, Clock Drawing Test, Frontal Assessment Battery, Corsi block-tapping task, Corsi supraspan learning task, Corsi Recall, and Trail Making Test (A, B, and A-B). An MRI was also performed and only subjects with normal results (i.e., no sign of white matter lesion or atrophy) were enrolled in the study. MRIs were performed within 6 months from subjects' enrollment. None of the controls had a history of neurological disorders, head trauma with loss of consciousness, epilepsy, brain surgery, systemic illness, or excessive drug or alcohol consumption at any time during their life. Participants were instructed not to drink any beverages containing caffeine or alcohol during the $24 \mathrm{~h}$ before the experiment. The local institutional review board approved the study.

Task and experimental design. The motor tasks have been extensively described in previous papers (Ghilardi et al., 2000; Huber et al., 2004; Marinelli et al., 2009). Briefly, subjects moved a cursor with their dominant or nondominant hand on a digitizing tablet and performed outand-back movements toward one of eight targets presented on the computer screen every $1.5 \mathrm{~s}$. Vision of both the hand and arm was prevented by an opaque panel, but the position of the cursor on the screen was always visible. Targets were positioned $4 \mathrm{~cm}$ from a common starting point and were presented in blocks of 48 (block duration, $72 \mathrm{~s}$ ). Hand trajectory was sampled at $200 \mathrm{~Hz}$.

We used two tasks: a baseline motor task, in which the cursor position on the screen and the hand position on the tablet corresponded; and a visuomotor adaptation task (ROT), in which the cursor position on the screen was rotated $30^{\circ}$ counterclockwise to the actual hand movement.

In the main experiment, after familiarization with the apparatus, subjects performed two blocks of baseline condition with each hand; no visual distortion was applied. Subsequently, they performed 10 blocks of ROT with the dominant (right) hand, followed by three blocks of ROT with the nondominant (left) hand.

Three weeks later, seven patients (six women; median age, 50 years; range, 39-57 years) and eight normal controls (five women; median age, 58.5 years; range, $42-66$ years) were retested, in the same clinical conditions, with one block ( 48 movements) of the baseline motor task performed with the left hand to assess long-term aftereffects.

Behavioral data analysis. In this study, we were interested in describing the process of adaptation to the imposed cursor distortion. Therefore, we first measured the initial planning of the movement direction as directional error at peak velocity, which is the difference between the target direction and the movement direction at peak velocity. For each hand, directional errors in the ROT blocks were normalized (DirErrVnorm) by subtracting the mean error in the corresponding baseline motor task where no rotation was applied.

We then computed the average percentage adaptation to the applied distortion for each ROT block as: percentage adaptation $=100 *(1-$ DirErrVnorm/30). We then measured the movement curvature to investigate the degree of online correction as the absolute difference between the directional error at peak velocity and that at the reversal point.

To measure the degree of total adaptation achieved with the right hand, we computed the difference in percentage adaptation between the
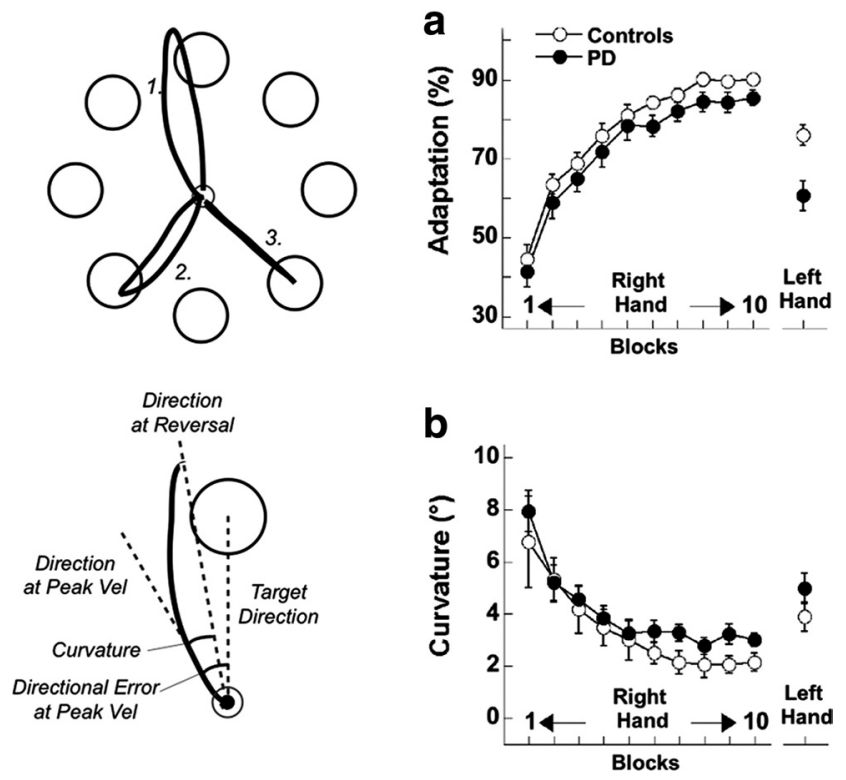

Figure 1. Top left, Target array with three trajectories representative of initial (1), intermediate (2), and complete (3) levels of adaptation. Bottom left, Illustration of directional error and curvature at an initial level of adaptation (see line 1, top left panel). $\boldsymbol{a}, \boldsymbol{b}$, Time course of adaptation (based on directional errors at the peak velocity; $\boldsymbol{a}$ ) and curvature $(\boldsymbol{b})$ as a function of blocks for the right hand and for transfer to the left hand in patient and control groups. Bars represent SEs.

Table 1. Anatomic locations with side, spatial extent of cluster in voxels (Ke), $t$ score, and Z score of striatal areas showing significant values, MNI coordinates

MNI coordinates
Side Cluster size tvalue Zscore $(x, y, z)$

Two-samples $t$ test: PD versus HC

$\begin{array}{lllrlc}\text { Putamen } & \text { R } & 1844 & 13.56 & 6.95 & 32,0,0 \\ \text { Caudate } & \text { R } & \text { Subcluster } & 9.58 & 5.95 & 16,12,10 \\ \text { Ventral striatum } & \text { R } & \text { Subcluster } & 4.95 & 4.61 & 18,20,-6 \\ \text { Putamen } & \mathrm{L} & 1575 & 11.14 & 6.39 & -28,-6,-2 \\ \text { Caudate } & \mathrm{L} & \text { Subcluster } & 6.58 & 4,84 & -14,20,-8\end{array}$

Covariance analysis of adaption transfer score

\begin{tabular}{lrrrrl} 
Caudate & R & 76 & 3.7 & 3.23 & $16,8,14$ \\
Putamen & R & 219 & 3.0 & 2.71 & $32,4,-2$ \\
\hline
\end{tabular}

L, Left; R, right.

first and last block. The same difference was computed for the movement curvature.

Right-to-left interlimb transfer was measured as the percentage difference between the last adaptation block with the right hand and the first with the left hand for both indices.

We assessed differences in the rate of adaptation with the right hand, as well as changes in curvature, by performing a mixed-model ANOVA ( $\alpha=0.05)$ on both variables with Group (PD, controls) as the betweensubject factor and Block (1-10) as the within-subject factor.

The between-group differences in the degree of total adaptation achieved and the difference in curvature, as well as for the interlimb transfer of both these variables, were assessed using a one-way ANOVA with Group as main factor.

SPECT data acquisition and reconstruction. SPECT data acquisition and reconstruction has been described in detail previously (Isaias et al., 2010). In brief, dopamine-transporter (DAT) values were measured with SPECT with FP-CIT. Intravenous administration of 110-185 MBq of ${ }^{123}$ I-FP-CIT (DaTSCAN; GE Healthcare) was performed 30-40 min after thyroid blockade (10-15 mg of Lugol solution per os) in all patients. Data were compared with data from a group of 15 healthy subjects matched for age (mean age, 62 years; \pm 9 SD; range, $44-68$ years) and sex 
Table 2. DAT binding values

\begin{tabular}{lllll}
\hline & CN right & CN left & PT right & PT left \\
\hline PD & $1.21 \pm 0.16(0.99-1.51)^{*}$ & $1.2 \pm 0.18(0.99-1.46)^{*}$ & $1.74 \pm 0.26(1.2-2.1)^{*}$ & $1.92 \pm 0.32(1.47-2.42)^{*}$ \\
HC & $1.77 \pm 0.29(1.15-2.1)$ & $1.59 \pm 0.33(1.19-2.04)$ & $2.54 \pm 0.32(1.85-3.04)$ & $2.79 \pm 0.35(2.06-3.47)$ \\
\hline
\end{tabular}

Subjects with PD showed a significant reduction of DAT binding values in both the caudate nucleus (CN) and putamen (PT) bilaterally when compared to a group of $15 \mathrm{HC}$. Data are reported as mean \pm SD and range (in brackets). ${ }^{*} p<0.001$.
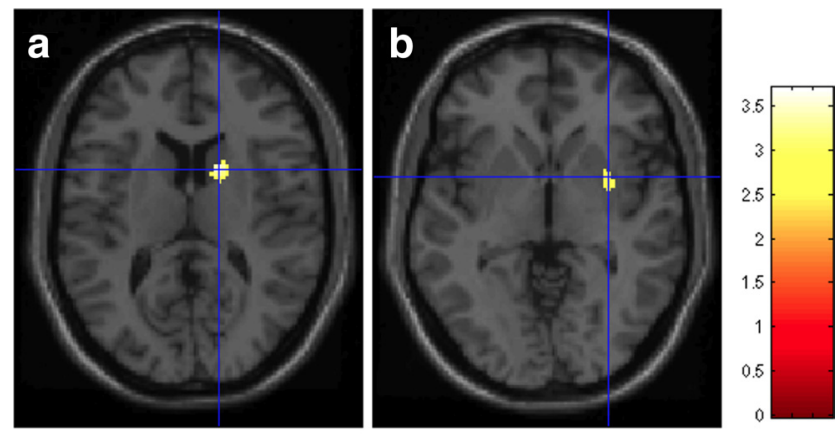

C
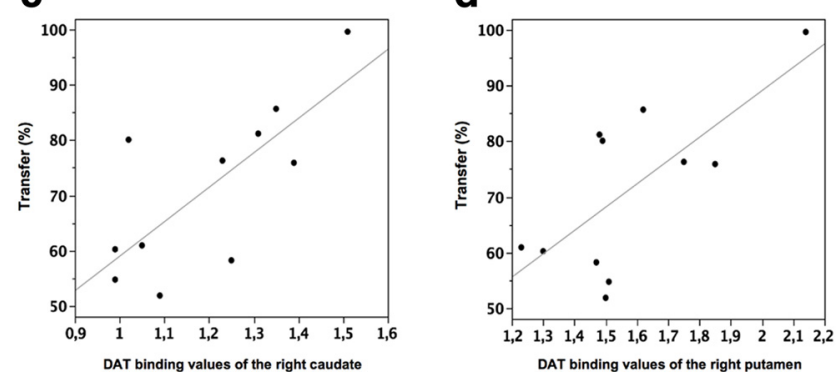

Figure 2. $\quad \boldsymbol{a}, \boldsymbol{b}$, Whole-brain voxelwise statistical analysis (SPM) showing a positive correlation between transfer of adaptation and DAT density in the right caudate $(x, y, z: 16,8,14)$ and right putamen $(x, y, z: 32,4,-2)$. c, $\boldsymbol{d}$, A volume of interest analysis confirming a positive correlation for the right caudate nucleus $(\rho=0.74, p<0.01)$ and right putamen $(\rho=0.71$, $p=0.01)$.

(four males). Brain SPECT was performed 3-4 h later by means of a dedicated triple detector gamma-camera (Prism 3000; Philips) equipped with low-energy, ultra-high-resolution fan beam collimators (four subsets of acquisitions; matrix size, $128 \times 128$; radius of rotation, $12.9-13.9$ $\mathrm{cm}$; continuous rotation; angular sampling, $3^{\circ}$; duration, $28 \mathrm{~min}$ ) in patient and control groups. Brain sections were reconstructed with an iterative algorithm (ordered subset expectation maximization, four iterations and 15 subsets), followed by 3D filtering of sections obtained (Butterworth, order 5, cutoff $0.31 \mathrm{Ny}$ ) and attenuation correction (Chang method, factor 0.12).

Imaging data processing. The reconstructed images were analyzed for regionally specific FP-CIT binding using Statistical Parametric Mapping (SPM2; Wellcome Department of Imaging Neuroscience, London, UK) in conjunction with MATLAB version R2007a (Mathworks).

First, we created a group-specific ${ }^{123}$ I-FP-CIT SPECT template with SPM2 by spatially normalizing the FP-CIT images of 15 healthy subjects onto a ${ }^{18}$ F-FP-CIT PET MNI-based template as previously described (Ma et al., 2002; Isaias et al., 2010), averaging the normalized images and their symmetric (mirror) image and filtering using a 3D Gaussian kernel with $8 \mathrm{~mm}$ full width at half maximum (FWHM) (Kas et al., 2007). Then, the FP-CIT images of all subjects were spatially normalized onto this FP-CIT template and smoothed with a FWHM $10 \mathrm{~mm}$ Gaussian kernel to increase the signal-to-noise ratio and to account for subtle variations in anatomic structures. For each individual FP-CIT SPECT image, a parametric binding ratio image was calculated using the ImCalc toolbox in SPM. Binding values for each FP-CIT image were computed in a voxel-by-voxel manner [(voxel - occipital)/occipital]. The reference region in the occipital cortex was defined using the volume-of-interest of superior, middle, and inferior occipital gyri and calcarine gyri of the automated anatomical labeling (Tzourio-Mazoyer et al., 2002), using the Wake Forest University PickAtlas 2.4 software.

Voxelwise statistical analysis. A general linear model was used to perform the appropriate voxelwise statistics using SPM2 in whole brain of all subjects. The analysis was applied to SPECT images by means of singlesubject: conditions and covariates design. The PD patients and healthy controls were modeled as conditions and the covariates were the scores of acquisition and transfer for both adaptation and curvature. In addition, we performed a covariance analysis between the transfer scores and FPCIT SPECT images. In every analysis, we used no global normalization, no grand mean scaling, and threshold masking absolute of $>1$. For $t$ test in $\mathrm{PD}$ versus healthy controls (HC), $p<0.001$ with false discovery rate correction was considered significant and for covariance analysis, $p<$ 0.01 uncorrected was significant, both at clusters of at least 50 voxels. All coordinates are reported in MNI space.

General statistical analysis. Normality of data distribution was tested by the Shapiro-Wilks test. Gender distribution among groups was tested with $\chi$ square. Demographic data were compared by means of Wilcoxon two-group test. A multivariate pairwise correlation analysis was used to investigate statistical dependence among SPECT binding values demographic, clinical, and behavioral data. Statistical analyses were performed with the JMP statistical package, version 8.0.2 (SAS Institute).

\section{Results}

In the first 10 blocks, patients and controls gradually decreased their directional error at peak velocity and adapted to the imposed rotation at a similar rate (Block: $F_{(9,171)}=165.52, p<0.00001$; Group: $F_{(1,19)}=1.70, p=0.20$; Block $\times$ Group: $F_{(9,171)}=0.25, p=$ 0.99; Fig. 1a). The degree of total adaptation achieved was comparable in patients $(44.1 \% \pm 2.7 \%)$ and controls $(45.7 \% \pm$ $\left.2.9 \% ; F_{(1,19)}=0.14, p=0.71\right)$.

Both groups showed a similar and significant reduction in curvature across blocks (Block: $F_{(9,171)}=15.77, p<0.00001$; Group: $F_{(1,19)}=0.59, p=0.45$; Block $\times$ Group: $F_{(9,171)}=0.31$, $p=0.97$; Fig. $1 b)$, further indicating that adaptation indeed resulted from updating the internal model with reduced reliance on online corrections.

The transfer of adaptation to the performance with the left hand was lower in patients compared with controls (PD: $71.4 \pm$ 4.6 vs $\mathrm{HC}: 85.5 \pm 2.4$, mean $\left.\pm \mathrm{SE} ; F_{(1,19)}=5.82, p=0.03\right)$, while the transfer of curvature was similar in the two groups (PD: $2 \pm$ 0.6 vs HC: $1.9 \pm 0.6$, mean $\left.\pm \mathrm{SE} ; F_{(1,19)}=0.07, p=0.80\right)$.

In the subgroup of subjects tested 3 weeks later, we found that aftereffects were significantly larger in controls $\left(-7.49 \pm 3.40^{\circ}\right)$ than in patients with $\mathrm{PD}$, where such effects were virtually absent $\left(-0.29 \pm 3.78^{\circ} ; F_{(1,13)}=15.01 ; p=0.002\right)$.

A significant, although weak, correlation $(\rho=0.53, p<0.025)$ was found between the 3 week aftereffects and right-to-left hand transfer.

No correlation was found between behavioral and clinical or demographic data. In particular, we found no correlations between transfer of adaptation and UPDRS-III $(\rho=0.22 ; p=0.51)$, UPDRS-AK $(\rho=-0.18 ; p=0.58)$, and UPDRS-item 22 (rigidity score left: $\rho=-0.45, p=0.15$; rigidity score right: $\rho=0.15, p=$ 0.64 ), suggesting that the learning and transfer indices are not predicted by clinical signs of PD. In addition, transfer of adaptation did not correlate with age $(\rho=-0.33, p=0.31)$ or disease duration $(\rho=-0.06, p=0.84)$. 
Binding values are listed in Tables 1 and 2. On average, PD subjects showed a reduced DAT binding value of $32 \%$ in the right caudate, $25 \%$ in the left caudate, and 32\% in both right and left putamen. Average DAT binding loss was not statistically different between left and right striatum.

Whole-brain SPM analysis revealed a selective positive correlation between the transfer of adaptation, but not of curvature, and DAT binding values of the right caudate and putamen only (Fig. 2, $a$ and $b$, respectively). These results were confirmed by a volume of interest analysis (transfer of adaptation and right caudate: $\rho=0.74, p<0.01$; transfer of adaptation and right putamen: $\rho=0.71, p=0.01$; Fig. $2, c$ and $d$, respectively).

No correlation was found between the adaptation rate of the right hand and DAT binding of any brain areas.

\section{Discussion}

The main result of this study is that the transfer of visuomotor learning correlated with DAT binding of the right striatum, suggesting, for the first time, that the levels of striatal dopamine influence the degree of interlimb transfer of a visuomotor skill.

In agreement with previous studies with similar paradigms (Marinelli et al., 2009; Bédard and Sanes, 2011; Venkatakrishnan et al., 2011) or prism adaptation (Weiner et al., 1983; Stern et al., 1988), patients with PD adapted their movements to an applied visual distortion similarly to healthy subjects. This visuomotor learning occurred by decreasing both initial directional error and hand path curvature. The decrement of both measures suggests that adaptation was achieved by the progressive modification of the motor plan and not by the constant use of online corrections of the trajectory.

When we tested the left hand, PD patients showed a reduced transfer of visuomotor learning compared with controls. The degree of transfer positively correlated with the values of DAT binding of the right striatum, but was not predicted by the UPDRS scores. Therefore, the observed deficit in transfer cannot be ascribed to a disease-related motor impairment, but depends on the dopaminergic innervation itself.

The specific mechanisms underlying interlimb transfer of visuomotor adaptation are still largely unknown. Adapting to a visuomotor rotation is likely to involve both effector-independent and effector-specific processes. The former consist of the progressive creation of new mapping between the direction of the hand trajectory and of the target, expressed in visual coordinates and therefore independent of the characteristics of the actual effector (i.e., the limb). The latter consist of the selection and implementation of the appropriate dynamic motor commands that translate the new motor plan into the actual muscle-activation patterns, which are specific for the effector involved. Training with one hand, therefore, likely promotes the formation of both a visuospatial memory, which can be shared by different effectors, and an effector-specific visuomotor memory. Therefore, we can assume that interlimb transfer is possible as the untrained hand can access the newly formed visual mapping. The fact that transfer is not complete, at least at the beginning of a task, implies that the formation of an effector-specific memory is also essential to complete the adaptation process. The reduced amount of transfer that we found in patients with PD could be due to impairments in both the formation and retrieval of the new visuospatial map, as well as in the efficient conversion of such a map to the untrained arm. Of note, the clinical signs in our patients were bilateral and of comparable severity, as also shown by similar DAT binding loss in both right and left striatum. Therefore, the reduced interlimb transfer should not be ascribed to a differential impairment of the two limbs. In this and previous studies (Marinelli et al., 2009), we also found that PD patients display an impairment of the long-term retention of this visuomotor skill that, in the present patient population, is highly correlated with the degree of transfer. Thus, it is possible that the reduced transfer in PD is part of a general deficit in skill formation, retention, and retrieval. Despite adaptation rates within normal limits, it is possible that the formation of a stable memory trace is not effective in patients with $\mathrm{PD}$, thus impairing short- and long-term memory processes. Accordingly, animal studies suggested a central role of striatal dopaminergic innervation in skill formation (Graybiel, 1998) and strengthening of synaptic plasticity (Calabresi et al., 2000; Lovinger et al., 2003).

The correlation found between the magnitude of the transfer and the dopamine levels was confined to the right striatum. Such lateralization could be explained by the direction of the transfer, from the right to the left hand. However, other theories should consider the right-hemisphere dominancy for this task (Ghilardi et al., 2000; Huber et al., 2004; Seidler et al., 2008) and for visuospatial abilities in general (Heilman et al., 1986). Future studies on the transfer in the opposite direction (left hand toward right hand) could settle the question of hemispheric dominance in this task. So far, studies with our task (Ghilardi et al., 2000; Huber et al., 2004) or similar paradigms (Seidler at al. 2008) showed that activity in the right-hemisphere, and in particular in the right parietal cortex, is essential for the formation and the retention of a new visuospatial mapping. Retrieval of the learned visuospatial skill could thus involve both cortical and striatal structures on the right. It is important to note that this study aimed at specifically examining the role of dopamine in interlimb transfer and not the related anatomical network. Future studies will investigate whether such a transfer relies on transcallosal projections from the cortex (e.g., right parietal cortex) to the ipsilateral and contralateral striatum, or a direct interstriatal transfer through the anterior commissure (Steiner et al., 1985).

\section{References}

Bédard P, Sanes JN (2011) Basal ganglia-dependent processes in recalling learned visual-motor adaptations. Exp Brain Res 209:385-393.

Calabresi P, Gubellini P, Centonze D, Picconi B, Bernardi G, Chergui K, Svenningsson P, Fienberg AA, Greengard P (2000) Dopamine and cAMPregulated phosphoprotein $32 \mathrm{kDa}$ controls both striatal long-term depression and long-term potentiation, opposing forms of synaptic plasticity. J Neurosci 20:8443-8451.

Calabresi P, Picconi B, Tozzi A, Di Filippo M (2007) Dopamine-mediated regulation of corticostriatal synaptic plasticity. Trends Neurosci 30:211-219.

Ghilardi MF, Alberoni M, Rossi M, Franceschi M, Mariani C, Fazio F (2000) Visual feedback has differential effects on reaching movements in Parkinson's and Alzheimer's disease. Brain Res 876:112-123.

Graybiel AM (1998) The basal ganglia and chunking of action repertoires. Neurobiol Learn Mem 70:119-136.

Halsband U, Lange RK (2006) Motor learning in man: a review of functional and clinical studies. J Physiol Paris 99:414-424.

Heilman KM, Bowers D, Valenstein E, Watson RT (1986) The right hemisphere: neuropsychological functions. J Neurosurg 64:693-704.

Huber R, Ghilardi MF, Massimini M, Tononi G (2004) Local sleep and learning. Nature 430:78-81.

Isaias IU, Benti R, Cilia R, Canesi M, Marotta G, Gerundini P, Pezzoli G, Antonin A (2007) [ $\left.{ }^{123} \mathrm{I}\right] \mathrm{FP}-\mathrm{CIT}$ striatal binding in early Parkinson's disease patients with tremor vs. akinetic-rigid onset. Neuroreport 18:1499-1502.

Isaias IU, Marotta G, Hirano S, Canesi M, Benti R, Righini A, Tang C, Cilia R, Pezzoli G, Eidelberg D, Antonini A (2010) Imaging essential tremor. Mov Disord 25:679-686.

Karabanov A, Cervenka S, de Manzano O, Forssberg H, Farde L, Ullén F (2010) Dopamine D2 receptor density in the limbic striatum is related to implicit but not explicit movement sequence learning. Proc Natl Acad Sci U S A 107:7574-7579. 
Kas A, Payoux P, Habert MO, Malek Z, Cointepas Y, El Fakhri G, ChaumetRiffaud P, Itti E, Remy P (2007) Validation of a standardized normalization template for statistical parametric mapping analysis of ${ }^{123} \mathrm{I}$-FP-CIT images. J Nucl Med 48:1459-1467.

Knowlton BJ, Mangels JA, Squire LR (1996) A neostriatal habit learning system in humans. Science 273:1399-1402.

Krakauer JW (2009) Motor learning and consolidation: the case of visuomotor rotation. Adv Exp Med Biol 629:405-421.

Lovinger DM, Partridge JG, Tang KC (2003) Plastic control of striatal glutamatergic transmission by ensemble actions of several neurotransmitters and targets for drugs of abuse. Ann N Y Acad Sci 1003:226-240.

Ma Y, Dhawan V, Mentis M, Chaly T, Spetsieris PG, Eidelberg D (2002) Parametric mapping of [18F]FPCIT binding in early stage Parkinson's disease: a PET study. Synapse 45:125-133.

Maia TV (2009) Reinforcement learning, conditioning, and the brain: successes and challenges. Cogn Affect Behav Neurosci 9:343-364.

Marinelli L, Crupi D, Di Rocco A, Bove M, Eidelberg D, Abbruzzese G, Ghilardi MF (2009) Learning and consolidation of visuomotor adaptation in Parkinson's disease. Parkinsonism Relat Disord 15:6-11.

Montague PR, Hyman SE, Cohen JD (2004) Computational roles for dopamine in behavioural control. Nature 431:760-767.

Sainburg RL, Wang J (2002) Interlimb transfer of visuomotor rotations: independence of direction and final position information. Exp Brain Res $145: 437-447$

Seidler RD, Noll DC (2008) Neuroanatomical correlates of motor acquisition and motor transfer. J Neurophysiol 99:1836-1845.

Seidler RD, Noll DC, Chintalapati P (2006) Bilateral basal ganglia activation associated with sensorimotor adaptation. Exp Brain Res 175:544-555.

Steiner H, Morgan S, Huston JP (1985) Effect of forebrain commissurotomy on recovery from unilateral 6-OHDA lesions of the substantia nigra and circling induced by apomorphine. Behav Brain Res 17:245-249.

Stern Y, Mayeux R, Hermann A, Rosen J (1988) Prism adaptation in Parkinson's disease. J Neurol Neurosurg Psychiatry 51:1584-1587.

Sutton RS, Barto AG (1998) Reinforcement learning: an introduction. Cambridge, MA: MIT.

Tzourio-Mazoyer N, Landeau B, Papathanassiou D, Crivello F, Etard O, Delcroix N, Mazoyer B, Joliot M (2002) Automated anatomical labeling of activations in SPM using a macroscopic anatomical parcellation of the MNI MRI single-subject brain. Neuroimage 15:273-289.

Venkatakrishnan A, Banquet JP, Burnod Y, Contreras-vidal JL (2011) Parkinson's disease differentially affects adaptation to gradual as compared to sudden visuomotor distortions. Hum Mov Sci 30:760-769.

Weiner MJ, Hallett M, Funkenstein HH (1983) Adaptation to lateral displacement of vision in patients with lesions of the central nervous system. Neurology 33:766-772. 